\title{
Locally Finite Functional Polyadic Algebra with Terms
}

\author{
Kahtan.H. Alzubaidy, Nabila.M. Bennour \\ Mathematics Department, Faculty of Science, Garyounis University
}

Keywords: Monadic Algebra; Polyadic Algebra; Terms.

Abstract: In 1956 P.R.Halmos [3] introduced polyadic algebra to express first order logic algebraically. In this work we extend polyadic algebra to include terms as

\section{INTRODUCTION}

The extension of Boolean algebra to several variable functional Boolean algebra with certain operators is capable of expressing polyadic logic [1] in an algebraic form called polyadic algebra [3] The operators correspond to the usual existential and universal quantifiers. The main issue of this paper is the study of terms as an embedded part of the system of polyadic algebra. Our study case is the locally finite functional polyadic algebra over a countable number of variables. The importance of functional case stems from the fact that any locally finite polyadic algebra is isomorphic to a functional polyadic algebra [3]. At the end of the paper a suggestion for the general case of locally finite polyadic algebra with terms is made.

\section{POLYADIC ALGEBRA}

\section{General definition [3]:}

Suppose that $B$ is a complete Boolean algebra. An existential quantifier on $B$ is a mapping $\exists: B \rightarrow B$ such that

i) $\exists(0)=0$

ii) $a \leq \exists(a)$ for any $a \in B$.

iii) $\exists(a \wedge \exists(b))=\exists(a) \wedge \exists(b)$ for any $a, b \in B$.

$(B, \exists)$ is called monadic algebra.

Let $I$ be a set usually countable to index the variables. A mapping not necessarily one to one or onto from $I$ into itself is called a transformation.

Let $I^{I}=\{\tau \mid \tau: I \rightarrow I$ is a transformation $\}$ Denote the set of all endomorphisms on $B$ by $\operatorname{End}(B)$ and the set of all quantifiers on $B$ by $\operatorname{Qant}(B)$.

A polyadic algebra is $(B, I, S, \exists)$ where $S: I^{I} \rightarrow \operatorname{End}(B)$ and $\exists: 2^{I} \rightarrow \operatorname{Qant}(B)$ such that for any $J, K \in 2^{I}$ and $\sigma, \tau \in I^{I}$ we have

1) $\exists(\phi)=i d$

3) $S(\sigma)=S(\sigma) S(\tau)$

2) $\exists(J \cup K)=\exists(J) \exists(K)$

5) $S(\sigma) \exists(J)=S(\tau) \exists(J)$ if $\left.\sigma\right|_{I-J}=\left.\tau\right|_{I-J}$

6) $\exists(J) S(\tau)=S(\tau) \exists\left(\tau^{-1}(J)\right)$ if $\tau$ is one to one on $\tau^{-1}(J)$. The cardinal number $|I|$ is called the degree of the algebra. If $|I|=n$ we get the so called $n$-adic algebra. If $I=\phi$ then $2^{I}=\{\phi\}$ and $I^{I}=\{i d\}$. Therefore we get only the identity quantifier $\exists(\phi)=i d$. Thus 0 -adic algebra is just the Boolean algebra $B$. If $|I|=1$ then $I^{I}=\{i d\}$. Therefore there are only two quantifiers $\exists(\phi)$ and $\exists(I)$. Thus 1-adic algebra is the monadic algebra.

\section{Local Finiteness:}

A simplified version of polyadic algebra has been provided for a locally finite polyadic algebra [2]. It is done as follows: 
Let $i, j \in I$. Define a function $(i / j): I \rightarrow I$ by $(i / j)(k)=k$ for any $k \neq i$ and $(i / j)(i)=j .(i / j)$ is called a replacement function. A transformation $\tau: I \rightarrow I$ is called finite if there is a finite subset $J$ of $B$ such that $\left.\tau\right|_{I-J}=i d$. It is well known that any finite transformation on $\mathrm{I}$ is a finite product of replacements [3]. Denote $S(i / j)$ by $(i / j)_{*}$. Let $a \in B$ and $J \subseteq I$ is called independent of $J$ if $\exists(J)(a)=a$. We say that $J$ is support a if a is independent of $I-J$ i.e $\exists(I-J)(a)=a$. A polyadic algebra is called locally finite if for any $a \in B$ there is a finite subset a $J$ of $I$ such that a $J$ supports $a$. In particular when $I$ is finite $B$ is locally finite.

Now any locally finite polyadic algebra $B$ is characterized as follows:

Theorem 1

For any $a \in B$ there is a finite subset a $J$ of $I$ such that the system $\left(B, I,\left\{(i / j)_{*}\right\},\left\{\exists_{i}\right\}\right), i, j \in J_{a}$ satisfies the following conditions:
i) $(i / j)_{*}=i d$
ii) $(i / k)_{*}(k / j)_{*}=(i / j)_{*}$
iii) $\exists(\phi)=i d$
iv) $\exists_{i} \exists_{j}=\exists_{j} \exists_{i}$
v) $\exists_{i}(i / j)_{*}=(i / j)_{*} \exists_{i}$

\section{FUNCTIONAL POLYADIC ALGEBRA}

Le $B=\left(B, \vee, \wedge,{ }^{\prime}, 0,1\right)$ be a complete Boolean algebra, $B^{A}=\{p \mid p: A \rightarrow B$ is a function $\}$ where $A$ is an algebra of type $\mathcal{F}$. For $p, q \in B^{A}$ define $p \vee q, p \wedge q, p^{\prime}$, and 0,1 pointwise as follows: $(p \vee q)(a)=p(a) \vee q(a),(p \wedge q)(a)=p(a) \wedge q(a), p^{\prime}(a)=(p(a))^{\prime}, 0(a)=0,1(a)=1$ for any $a \in A$. We have

Proposition 2 [2]

$\left(B^{A}, \vee, \wedge,{ }^{\prime}, 0,1\right)$ is a functional Boolean algebra.

Proposition 3 [2]

$\left(B^{A}, \exists\right)$ is a monadic algebra, where $\exists: B^{A} \rightarrow B^{A}$ is given by $\exists(p)(a)=\sup \{p(a): a \in A\}$

Proposition 4 [2]

$\left(B^{A}, I, S, \exists\right)$ is a polyadic algebra, where $S: I^{I} \rightarrow \operatorname{End}\left(B^{A}\right)$ and $\exists: 2^{I} \rightarrow \operatorname{Qant}\left(B^{A}\right)$

Now, let $B^{A^{k}}=\left\{p \mid p: A^{k} \rightarrow B\right.$ is a function $\} ; k=0,1,2, \ldots$. Define $S(\sigma) p\left(a_{1}, \ldots, a_{k}\right)=p\left(a_{\sigma(1)}, \ldots, a_{\sigma(k)}\right)$ for any $\sigma \in I^{I}$ and $\left(a_{1}, \ldots, a_{k}\right) \in A^{k}$. We have

Proposition 5

$\left(B^{A^{k}}, I, S, \exists\right)$ is a polyadic algebra

Proof

$$
\begin{aligned}
& \text { i) } \exists(\phi) p\left(a_{1}, \ldots, a_{k}\right)=\sup _{\phi}\left\{p\left(a_{1}, \ldots, a_{k}\right)\right\}=p\left(a_{1}, \ldots, a_{k}\right) \quad \therefore \exists(\phi)=i d \\
& \text { ii) } \exists(J \cup M)=\sup _{J \cup M}\left\{p\left(a_{1}, \ldots a_{k}\right)\right\} \\
& =\sup _{J}\left\{p\left(a_{1}, \ldots a_{k}\right)\right\} \sup _{K}\left\{p\left(a_{1}, \ldots, a_{k}\right)\right\} \\
& =\exists(J) p\left(a_{1}, \ldots, a_{k}\right) \exists(M) p\left(a_{1}, \ldots, a_{k}\right) \\
& \therefore \exists(J \cup M)=\exists(J) \exists(M) \\
& \text { iii) } S(i d) p\left(a_{1}, \ldots, a_{k}\right)=p\left(a_{i d(1)}, \ldots a_{i d(k)}\right)=p\left(a_{1}, \ldots, a_{k}\right) \quad \therefore S(i d)=i d \\
& \text { iv) } S(\sigma \pi) p\left(a_{1}, \ldots a_{k}\right)=p\left(a_{\sigma(1)}, \ldots, a_{\sigma(k)}\right)=p\left(a_{\sigma(\tau(1))}, \ldots, a_{\sigma(\tau(k))}\right) \\
& =S(\sigma) p\left(a_{\tau(1)}, \ldots, a_{\tau(k)}\right)=S(\sigma) S(\tau) p\left(a_{1}, \ldots, a_{k}\right) \\
& \therefore S(\sigma \pi)=S(\sigma) S(\tau)
\end{aligned}
$$


v) $S(\sigma) \exists(J) p\left(a_{1}, \ldots, a_{k}\right)=S(\sigma) \sup _{J}\left\{p\left(a_{1}, \ldots, a_{k}\right\}\right.$

$$
\begin{aligned}
& =\sup _{J}\left\{p\left(a_{\sigma(1)}, \ldots a_{\sigma(k)}\right)\right\} \\
& =\sup _{J}\left\{p\left(a_{\tau(1)}, \ldots, a_{\tau(k)}\right\} \text { if }\left.\sigma\right|_{I-J}=\left.\tau\right|_{I-J}\right. \\
& =S(\tau) \sup _{J}\left\{p\left(a_{1}, \ldots, a_{k}\right)\right\} \\
& =S(\tau) \exists(J) p\left(a_{1}, \ldots, a_{k}\right)
\end{aligned}
$$

$S(\sigma) \exists(J)=S(\tau) \exists(J) \quad$ if $\left.\sigma\right|_{I-J}=\left.\tau\right|_{I-J}$

vi) $\exists(J) S(\tau) p\left(a_{1}, \ldots, a_{k}\right)=\exists(J) p\left(a_{\tau(1)}, \ldots, a_{\tau(k)}\right)$

$$
\begin{aligned}
& =\sup _{J}\left\{p\left(a_{\tau(1)}, \ldots, a_{\tau(k)}\right)\right\} \quad \text { if } \tau \text { is } 1-1 \text { on } \tau^{-1}(J) \\
& =\sup _{\tau^{-1}(J)}\left\{p\left(a_{\tau(1)}, \ldots, a_{\tau(k)}\right)\right\} \\
& =\sup _{\tau^{-1}(J)}\left\{S(\tau) p\left(a_{1}, \ldots, a_{k}\right)\right\} \\
& =S(\tau) \exists\left(\tau^{-1}(J)\right) p\left(a_{1}, \ldots, a_{k}\right)
\end{aligned}
$$

$\therefore \exists(J) S(\tau)=S(\tau) \exists\left(\tau^{-1}(J)\right)$

The algebra $\left(B^{A^{k}}, I,\left\{(i / j)_{*}\right\},\left\{\exists_{i}\right\}\right)$ is locally finite, and hence it satisfies the following conditions:
i) $\quad(i / i)_{*}=i d$
ii) $(i / m)_{*}(\mathrm{~m} / \mathrm{j})_{*}=(i / j)_{*}$
iii) $\exists(\phi)=i d$
iv) $\exists_{i} \exists_{i}=\exists_{i} \exists_{i}$
v) $\exists_{i}(i / j)_{*}=(i / j)_{*} \exists_{i}$

They are satisfied as follows:

i) $\quad(i / i)_{*} p\left(a_{1}, \ldots, a_{k}\right)=S(i / i) p\left(a_{1}, \ldots, a_{k}\right)=p\left(a_{(i / i)(1)}, \ldots, a_{(i / i)(k)}\right)$

$$
=p\left(a_{i d(1)}, \ldots, a_{i d(k)}\right)=p\left(a_{1}, \ldots, a_{k}\right) \quad \therefore(i / i)_{*}=i d
$$

ii) $(i / m)_{*}(m / j)_{*} p\left(a_{1}, \ldots, a_{k}\right)=S(i / m) S(m / j) p\left(a_{1}, \ldots, a_{k}\right)$

$$
\begin{aligned}
& =S(i / m) p\left(a_{(m / j)(1}, \ldots, a_{(m / j)(k)}\right) \\
& =p\left(a_{(i / m)(m / j)(1}, \ldots, a_{(i / m)(m / j)(k)}\right) \\
& =p\left(a_{(i / j)(1)}, \ldots, a_{(i / j)(k)}\right) \\
& =S(i / j) p\left(a_{1}, \ldots, a_{k}\right)=(i / j)_{*} p\left(a_{1}, \ldots, a_{k}\right)
\end{aligned}
$$

$\therefore(i / m)_{*}(m / j)_{*}=(i / j)_{*}$

iii ) $\exists(\phi) p\left(a_{1}, \ldots, a_{k}\right)=\sup _{\phi}\left\{p\left(a_{1}, \ldots, a_{k}\right)\right\}=p\left(a_{1}, \ldots, a_{k}\right) \quad \therefore \exists(\phi)=i d$

iv) $\exists_{i} \exists_{j}(p)\left(a_{1}, \ldots, a_{k}\right)=\exists_{i}\left(\sup _{\{j\}}\left\{p\left(a_{1}, \ldots, a_{k}\right)\right\}\right)=\sup _{\{i\}}\left\{\sup _{\{j\}}\left\{p\left(a_{1}, \ldots, a_{k}\right)\right\}\right\}$

$$
=\sup _{\{j\}}\left\{\sup _{\{i\}}\left\{p\left(a_{1}, \ldots, a_{k}\right)\right\}\right\}=\exists_{j} \exists_{i}(p)\left(a_{1}, \ldots, a_{k}\right)
$$

$\therefore \exists_{i} \exists_{j}=\exists_{j} \exists_{i}$

v) $\exists_{i}(i / j)_{*} p\left(a_{1}, \ldots, a_{k}\right)=\sup _{\{i\}}\left\{S(i / j) p\left(a_{1}, \ldots, a_{k}\right)\right\}$

$$
\begin{aligned}
& \qquad S(i / j) \sup _{\{i\}}\left\{p\left(a_{1}, \ldots, a_{k}\right)\right\}=(i / j)_{*} \exists_{i}(p)\left(a_{1}, \ldots, a_{k}\right) \\
& \therefore \exists_{i}(i / j)_{*}=(i / j)_{*} \exists_{i}
\end{aligned}
$$

Finally, let $I=\{1,2, \ldots\}$ be a countable set, $B^{A^{I}}=\left\{p \mid p: A^{I} \rightarrow B\right.$ is a function $\}$,

$a \in A^{I} ; a: I \rightarrow A$ is a function. For $\sigma \in I^{I}, \sigma a$ is defined by $\sigma a(i)=a(\sigma(i)) \forall i \in I$.

We have 


\section{Proposition 6}

$B^{A^{I}}$ is a polyadic algebra

Proof

$$
\begin{aligned}
& \text { i) } \quad \exists(\phi) p(a)=\sup \{p(a)\}=p(a) \quad \therefore \exists(\phi)=i d \\
& \text { ii) } \exists(J \cup M) p(a)=\sup _{J \cup M}\{p(a)\}=\sup _{J}\{p(a)\} \sup _{M}\{p(a)\} \\
& =\exists(J) p(a) \exists(M) p(a) \quad \therefore \exists(J \cup M)=\exists(J) \exists(M) \\
& \text { iii) } S(\text { id }) p(a(i))=p(a(i d(i)))=p(a(i)) \\
& \therefore S(i d)=i d \\
& \text { iv) } S(\sigma \tau) p(a(i))=p(a \sigma(\tau(i)))=p(\sigma a(\tau(i))) \\
& =S(\sigma) p(\tau a(i))=S(\sigma) S(\tau) p(a(i)) \quad \therefore S(\sigma \tau)=S(\sigma) S(\tau) \\
& \text { v) } \quad S(\sigma) \exists(J) p(a(i))=S(\sigma) \sup _{J}\{p(a(i))\}=\sup _{J}\{p(a \sigma(i))\} \\
& =\sup _{J}\{p(a \tau(i))\} \quad \text { if }\left.\sigma\right|_{I-J}=\left.\tau\right|_{I-J} \\
& =S(\tau) \sup _{J}\{p(a(i))\}=S(\tau) \exists(J) p(a(i)) \\
& \therefore S(\sigma) \exists(J)=S(\tau) \exists(J) \\
& =\sup _{\tau^{-1}(J)}\{p(a \tau(i))\} \text { if } \tau \text { is } 1-1 \text { on } \tau^{-1}(J) \\
& =\sup _{\tau^{-1}(J)}\{S(\tau) p(a(i))\}=S(\tau) \sup _{\tau^{-1}(J)}\{p(a(i))\} \\
& =S(\tau) \exists\left(\tau^{-1}(J)\right) p(a(i)) \\
& \therefore \exists(J) S(\tau)=S(\tau) \exists\left(\tau^{-1}(J)\right)
\end{aligned}
$$

The algebra $\left(B^{A^{I}}, I,\left\{(i / j)_{*}\right\},\left\{\exists_{i}\right\}\right)$ is also locally finite and hence it satisfies the following conditions
i) $(i / i)_{*}=i d$
ii) $(i / m)_{*}(m / j)_{*}=(i / j)_{*}$
iii) $\exists(\phi)=i d$
iv) $\exists_{i} \exists_{j}=\exists_{j} \exists_{i}$
v) $\exists_{i}(i / j)_{*}=(i / j)_{*} \exists_{i}$

They are satisfied as follows:

i) $(i / j)_{*} p(a(i))=S(i / j) p(a(i))=p(a(i / i)(i))=p(a(i)) \quad \therefore(i / i)_{*}=i d$

ii) $(i / m)_{*}(m / j)_{*} p(a(i))=S(i / m) S(m / j) p(a(i))=S(i / m) p(a(m / j)(i))$

$$
\begin{aligned}
& =p(a(i / m)(m / j)(i))=p(a(i / j)(i))=S(i / j) p(a(i)) \\
& =(i / j)_{*} p(a(i)) \quad \therefore(i / m)_{*}(m / j)_{*}=(i / j)_{*}
\end{aligned}
$$

iii) $\exists(\phi) p(a(i))=\sup _{\phi}\{p(a(i))\}=p(a(i))$

$$
\therefore \exists(\phi)=i d
$$

iv) $\exists_{i} \exists_{j} p(a(i))=\sup _{\{i\}}\left\{\sup _{\{j\}}\{p(a(i))\}\right\}=\sup _{\{j\}}\left\{\sup _{\{i\}}\{p(a(i)))\right\}=\exists_{j} \exists_{i} p(a(i))$

$\therefore \exists_{i} \exists_{j}=\exists_{j} \exists_{i}$

v) $\exists_{i}(i / j)_{*} p(a(i))=\sup _{\{i\}}\{S(i / j) p(a(i))\}=S(i / j) \sup _{\{i\}}\{p(a(i))\}=(i / j)_{*} \exists_{i} p(a(i))$

$\therefore \exists_{i}(i / j)_{*}=(i / j)_{*} p(a(i))$

\section{TERMS}

Let $X$ be a set of variables and $\mathcal{F}$ be a type of algebra. The set $T(X)$ of terms of type $\mathcal{F}$ over $X$ is the smallest set such that 
i) $X \cup \mathcal{F}_{0} \subseteq T(X)$

ii) If $t_{1}, \ldots, t_{n} \in T(X)$ and $f \in \mathcal{F}_{n}$ then $f\left(t_{1}, \ldots, t_{n}\right) \in T(X)$ [5]

For $t \in T(X)$ we often write $t$ as $t\left(x_{1}, \ldots, x_{n}\right)$ to indicate that the variables occurring in $t$ are among $x_{1}, \ldots, x_{n}$.

A term $t$ is n-ary if the number of variables appearing explicitly is less than or equal $\mathrm{n}$.

The term $t$ defines a function $t_{A}: A^{n} \rightarrow A$ as $t_{A}\left(a_{1}, \ldots, a_{n}\right)=t\left(x_{i} / a_{i}\right)$ for $a_{1}, \ldots, a_{n} \in A$

The set $T(X)$ can be transformed into an algebra [5]. The term algebra $T(X)$ of type $\mathcal{F}$ over $X$ has as its universe the set $T(X)$ and the fundamental operations satisfy :

$f^{T(X)}:\left(t_{1}, \ldots, t_{n}\right) \mapsto f\left(t_{1}, \ldots, t_{n}\right)$ for $f \in \mathcal{F}_{n}$ and $t_{i} \in T(X), \quad 1 \leq t \leq n$.

Now, consider the functional polyadic algebra $\left(B^{A}, I, S, \exists\right)$. A term $t$ defines a function

$t_{*}: B^{A} \rightarrow B^{A}$ as follows $t_{*}(p)(a)=p t_{A}(a)=p t(x / a)$

Thus obviously we have

Proposition 7
1) $t_{*} \in \operatorname{End}\left(B^{A}\right)$
2) $\exists t_{*}=t_{*} \exists$
3) $S(\sigma) t_{*}=t_{*} S(\sigma)$ for any $\sigma \in I^{I}$

Let $t=t\left(x_{1}, \ldots, x_{n}\right)$ be a term on the algebra $A$. By using finite compositions of product, projection, identity and /or constant functions on $\mathrm{x}_{1}, \mathrm{x}_{2}, \ldots, \mathrm{x}_{\mathrm{n}}$ the term $t$ can be transformed uniquely to a function, say again $t: A^{k} \rightarrow A^{k}$ when $n \leq k$ by $t=\left(t_{1}, t_{2}, \ldots, t_{k}\right)$ where each $t_{i}: A^{k} \rightarrow A$ is a function also for $i=1,2, \ldots, k$.

For $a=\left(a_{1}, \ldots, a_{k}\right) \in A^{k}$ and $p \in B^{A^{k}}$, let $t p(a)=p\left(t_{1}(a), \ldots, t_{k}(a)\right)$. This defines a mapping $t_{*}: B^{A^{k}} \rightarrow B^{A^{k}}$ given as follows:

$t_{*}(p)=p\left(t_{1}, \ldots, t_{k}\right)$ for any $p \in B^{A^{k}}$ where $t_{*}(p)(a)=p\left(t_{1}(a), \ldots, t_{k}(a)\right)$ for any $a \in A^{k}$.

Proposition 8)

$\begin{array}{ll}\text { 1) } t_{*} \in \operatorname{End}\left(B^{A^{k}}\right) & \text { 2) } t_{*} \exists(J)=\exists(J) t_{*} \text { for any } J \subseteq I\end{array}$

3) $S(\sigma) t_{*}=t_{*} S(\sigma)$ for any $\sigma \in I^{I}$

Proof

1) i) $t_{*}(p \vee q)(a)=(p \vee q)\left(t_{1}(a), \ldots, t_{k}(a)\right)$

$$
\begin{aligned}
& =p\left(t_{1}(a), \ldots, t_{k}(a)\right) \vee q\left(t_{1}(a), \ldots, t_{k}(a)\right) \\
& =t_{*}(p)(a) \vee t_{*}(q)(a)
\end{aligned}
$$

$\therefore t_{*}(p \vee q)=t_{*}(p) \vee t_{*}(q)$

ii) $t_{*}(p \wedge q)(a)=(p \wedge q)\left(t_{1}(a), \ldots, t_{k}(a)\right)$

$=p\left(t_{1}(a), \ldots, t_{k}(a)\right) \wedge q\left(t_{1}(a), \ldots, t_{k}(a)\right)$

$=t_{*}(p)(a) \wedge t_{*}(q)(a)$

$\therefore t_{*}(p \wedge q)=t_{*}(p) \wedge t_{*}(q)$

iii) $\left(t_{*}(p)(a)\right)^{\prime}=\left(p\left(t_{1}(a), \ldots, t_{k}(a)\right)\right)^{\prime}=p^{\prime}\left(t_{1}(a), \ldots, t_{k}(a)\right)=t_{*}(p)^{\prime}(a)$

$$
\therefore\left(t_{*}(p)\right)^{\prime}=t_{*}(p)^{\prime}
$$

iv) $t_{*}(0)(a)=0\left(t_{1}(a), \ldots, t_{k}(a)\right)=0=0(a) \quad \therefore t_{*}(0)=0$

v) $t_{*}(1)(a)=(1)\left(t_{1}(a), \ldots, t_{k}(a)\right)=\left(t_{1}(a), \ldots, t_{k}(a)\right)=t_{*}(a)$

$$
\therefore t_{*}(1)=t_{*}
$$

2) $t_{*} \exists(J)(p)(a)=t_{*}\left(\sup _{J}\{p(a)\}\right)=\sup _{J}\left\{p\left(t_{1}(a), \ldots, t_{k}(a)\right)\right\}$

$$
=\sup _{J}\left\{t_{*}(p)(a)\right\}=\exists(J) t_{*}(p)(a)
$$




$$
\text { 3) } \begin{aligned}
t_{*} S(\sigma) p(a) & =t_{*} p(a \sigma)=p\left(t_{1}(a \sigma), \ldots, t_{k}(a \sigma)\right)=p\left(t_{1}(a) \sigma, \ldots, t_{k}(a) \sigma\right) \\
& =S(\sigma) p\left(t_{1}(a), \ldots, t_{k}(a)\right)=S(\sigma) t_{*} p(a) \quad \therefore S(\sigma) t_{*}=t_{*} S(\sigma)
\end{aligned}
$$

Not that proposition 7 is a special case of proposition 8 .

Finally, consider the countable indexing set $I=\{1,2,3, \ldots\}$ of variables on $A$.

A term $\mathrm{t}$ in a function involving a finite number of such variables. As above $\mathrm{t}$ induces a mapping $t_{*}: B^{A^{I}} \rightarrow B^{A^{I}}$ given as follows:

Let $p \in B^{A^{I}}$.Let $t_{*}(p)=\left(p t_{i}\right)$ for finitely many $i \in I$.

For each $a \in A^{I}$, define $t_{*}(p)(a)=\left(p t_{i}(a)\right)$.

Proposition 9

1) $t_{*} \in \operatorname{End}\left(B^{A^{I}}\right)$

2) $t_{*} \exists(J)=\exists(J) t_{*}$ for any $J \subseteq I$.

3) $S(\sigma) t_{*}=t_{*} S(\sigma)$ for any $\sigma \in I^{I}$

Proof

1) i) $t_{*}(p \vee q)(a)=(p \vee q) t_{i}(a)=p t_{i}(a) \vee q t_{i}(a)=t_{*}(p)(a) \vee t_{*}(p)(a)$

$$
\therefore t_{*}(p \vee q)=t_{*}(p) \vee t_{*}(q)
$$

ii) $t_{*}(p \wedge q)(a)=(p \wedge q) t_{i}(a)=p t_{i}(a) \wedge q t_{i}(a)=t_{*}(p)(a) \wedge t_{*}(p)(a)$

$\therefore t_{*}(p \wedge q)=t_{*}(p) \wedge t_{*}(q)$

iii) $\left(t_{*}(p)(a)\right)^{\prime}=\left(p t_{i}(a)\right)^{\prime}=p^{\prime} t_{i}(a)=t_{*}(p)^{\prime}(a)$

$\therefore\left(t_{*}(p)\right)^{\prime}=t_{*}(p)^{\prime}$

iv) $t_{*}(0)(a)=0 t_{i}(a)=0(a) \quad \therefore t_{*}(0)=0$

v) $t_{*}(1)(a)=(1) t_{i}(a)=t_{*}(a) \quad \therefore t_{*}(1)=t_{*}$

2) $t_{*} \exists(J) p(a)=t_{*} \sup _{J}\{p(a)\}=\sup _{J}\{p(a)\} t_{i}(a)=\sup _{J}\left\{t_{*}(p)(a)\right\}=\exists(J) t_{*}(p)(a)$

$$
\therefore t_{*} \exists(J)=\exists(J) t_{*}
$$

3) $t_{*} S(\sigma) p(a)=t_{*} p(a \sigma)=p\left(t_{i}(a \sigma)\right)=p\left(\left(t_{i}(a)\right) \sigma\right)=S(\sigma) p t_{i}(a)=S(\sigma) t_{*} p(a)$

$\therefore S(\sigma) t_{*}=t_{*} S(\sigma)$

Now it is possible to introduce a locally finite functional polyadic algebra with terms by $\left(B^{A^{I}}, I, S, \exists, T(X)\right)$ where $\mathrm{B}$ is a complete Boolean algebra, $A$ any algebra of type $\mathcal{F}, I$ a set usually countable to index the variables, $S: I^{I} \rightarrow \operatorname{End}\left(B^{A^{I}}\right)$, where $I^{I}=\{\tau \mid \tau: I \rightarrow I$ is a transformation $\}, \quad E n d\left(B^{A^{I}}\right)$ is the set of all endomorphisms on $B^{A^{I}}$, $\exists: 2^{I} \rightarrow \operatorname{Qant}\left(B^{A^{I}}\right)$, where $\operatorname{Qant}\left(B^{A^{I}}\right)$ is the set of all quantifiers on $B^{A^{I}}$ and $T(X)$ is the algebra of terms of $X$ where $X$ is the set of variables. The following conditions are hold:

i) $\exists(\phi)=i d$

ii) ii) $\exists(I \cup J)=\exists(I) \exists(J)$

iii) $\quad S(\sigma \tau)=S(\sigma) S(\tau)$

iv) $S(i d)=i d$

v) $S(\sigma) \exists(J)=S(\tau) \exists(J)$ if $\left.\sigma\right|_{I-J}=\left.\tau\right|_{I-J}$

vi) $\exists(J) S(\tau)=S(\tau) \exists\left(\tau^{-1}(J)\right)$ if $\tau$ is one to one on $\tau^{-1}(J)$.

vii) $t_{*} \in \operatorname{End}\left(B^{A^{I}}\right)$

viii) $t_{*} \exists(J)=\exists(J) t_{*}$

ix) $S(\sigma) t_{*}=t_{*} S(\sigma)$.

The first six conditions above may be replaced by the simpler five conditions of locally finite case as before. 


\section{GENERAL CASE}

One may suggest a system for the general locally finite polyadic algebra with terms. The system is $(B, I, S, \exists, T, F)$ which consists of:

1) a complete Boolean algebra $B$,

2) a countable set $I$ to index the variables,

3) a mapping $S: I \operatorname{End}(B) I \rightarrow$ where $\operatorname{End}(B)$ is the set of all endomorphisms of $B$,

4) a mapping: $2 \operatorname{Qant}(B) I \exists \rightarrow$ where $\operatorname{Qant}(B)$ is the set of all existential quantifiers on $B$,

$5)$ a set $T$ of terms on the variables, and

6) a mapping $F: T \rightarrow E n d(B)$ such that the following conditions hold:

i) $\exists(\phi=$ id

ii) $\exists(I \cup J)=\exists(I) \exists(J)$

iii) $\mathrm{S}(\sigma \tau)=\mathrm{S}(\sigma) \mathrm{S}(\tau)$

iv) $\mathrm{S}(\mathrm{id})=\mathrm{id}$

v) $\mathrm{S}(\sigma) \exists(\mathrm{J})=\mathrm{S}(\tau) \exists(\mathrm{J})$ if $\left.\right|_{I-J}=\left.\tau\right|_{I-J}$

vi) $\quad \exists(J) S(\tau)=S(\tau) \exists\left(\tau^{-1}(J)\right)$ if $\tau$ is one to one on $\tau^{-1}(J)$

vii) $F(t) \exists(J)=\exists(J) F(t)$

viii) $\mathrm{F}(\mathrm{t}) \mathrm{S}(\sigma)=\mathrm{S}(\sigma) \mathrm{F}(\mathrm{t})$.

\section{REFERENCES}

[1] A. G. Hamilton, Logic for Mathematicians. Cambridge University Press, Cambridge, (1988).

[2] K.H. Alzubaidy, On Algebraization Of Polyadic Logic. Journal of Mathematical Sciences, 18(1), 15-18 (2007).

[3] P. R. Halmos, Algebraic Logic II .Homogenous Locally Finite Polyadic Boolean Algebras Of Infinite Degree. Fundamenta Mathematicae 43, 255-325 (1956).

[4] P. R. Halmos, Lectures on Boolean Algebra. D.Van Nostran, Princeton, (1963).

[5] S. Burris \& H. P. Shakappanavor, A Course in Universal Algebra. New York: Springer-Verlag (1981). 